TRANSACTIONS OF THE

AMERICAN MATHEMATICAL SOCIETY

Volume 360, Number 12, December 2008, Pages 6319-6329

S 0002-9947(08)04597-2

Article electronically published on July 24, 2008

\title{
ISOMORPHISM RIGIDITY OF COMMUTING AUTOMORPHISMS
}

\author{
SIDDHARTHA BHATTACHARYA
}

\begin{abstract}
Let $d>1$, and let $(X, \alpha)$ and $(Y, \beta)$ be two zero-entropy $\mathbb{Z}^{d}$-actions on compact abelian groups by $d$ commuting automorphisms. We show that if all lower rank subactions of $\alpha$ and $\beta$ have completely positive entropy, then any measurable equivariant map from $X$ to $Y$ is an affine map. In particular, two such actions are measurably conjugate if and only if they are algebraically conjugate.
\end{abstract}

\section{INTRODUCTION}

It is well known that ergodic automorphisms of compact abelian groups are measurably isomorphic with Bernoulli shifts (cf. e.g. [14]). In particular, entropy is a complete measurable conjugacy invariant for such automorphisms. On the other hand, for $d>1$, mixing zero-entropy $\mathbb{Z}^{d}$-actions on compact abelian groups by $d$ commuting automorphisms tends to exhibit remarkable rigidity properties. In this paper we study rigidity of the measurable structure of these actions.

Before stating our main result, we recall a few basic definitions. Throughout this paper the term compact abelian group will denote an infinite compact metrizable abelian group. An algebraic $\mathbb{Z}^{d}$-action $(X, \alpha)$ is an action $\alpha$ of $\mathbb{Z}^{d}$ on a compact abelian group $X$ by continuous automorphisms. Any such action preserves $\lambda_{X}$, the normalized Haar measure on $X$. A lower rank subaction of $\alpha$ is the restriction of $\alpha$ to a subgroup $\Lambda \subset \mathbb{Z}^{d}$ with $\operatorname{rank}(\Lambda)<d$. If $(X, \alpha)$ and $(Y, \beta)$ are algebraic $\mathbb{Z}^{d}$ actions, then a Borel map $\phi: X \rightarrow Y$ is said to be equivariant if $\phi \circ \alpha(\mathbf{n})=\beta(\mathbf{n}) \circ \phi$ $\lambda_{X}$-a.e., for every $\mathbf{n} \in \mathbb{Z}^{d}$. The actions $\alpha$ and $\beta$ are measurably or algebraically conjugate if the map $\phi$ can be chosen to be a Borel isomorphism or a continuous group isomorphism. A map $\psi: X \rightarrow Y$ is affine if there exist a continuous group homomorphism $\psi^{\prime}: X \rightarrow Y$ and an element $y \in Y$ such that $\psi(x)=\psi^{\prime}(x)+y$ almost everywhere with respect to $\lambda_{X}$.

In this paper we prove the following theorem:

Theorem 1.1. Let $d>1$, and let $(X, \alpha)$ and $(Y, \beta)$ be zero-entropy algebraic $\mathbb{Z}^{d}$ actions such that all lower rank subactions of $\alpha$ and $\beta$ have completely positive entropy. Then every measurable equivariant map $f: X \rightarrow Y$ is an affine map. In particular, two such actions are measurably conjugate if and only if they are algebraically conjugate.

Received by the editors November 6, 2006.

2000 Mathematics Subject Classification. Primary 37A35, 37A15.

Key words and phrases. Rigidity, commuting automorphisms, entropy.

(C)2008 American Mathematical Society Reverts to public domain 28 years from publication 
Several results in this direction were obtained in recent years. The case when both $X$ and $Y$ are zero-dimensional was studied in [3], 8] and [13. For a certain class of actions on connected groups with the property that the entropy of every individual element in $\mathbb{Z}^{d}$ is finite, in 9 and [12] the above result was proved as a consequence of more general results on invariant measures. In [10, Theorem 1.1 was proved for a class of $\mathbb{Z}^{3}$-actions on connected groups in which every individual element has infinite entropy.

The more general situation where $\alpha$ and $\beta$ are arbitrary mixing zero-entropy actions was studied in [1, 2] and [4. Although these actions also exhibit several rigidity properties, there are mixing zero-entropy algebraic $\mathbb{Z}^{d}$-actions on zerodimensional groups for which the analogue of Theorem 1.1 fails (cf. [1, 3]). It is not known whether one can construct similar examples when both $X$ and $Y$ are connected (cf. 20, p. 46).

\section{BACKGROUND}

Let $R_{d}=\mathbb{Z}\left[u_{1}^{ \pm 1}, \ldots, u_{d}^{ \pm 1}\right]$ be the ring of Laurent polynomials with integral coefficients in the commuting variables $u_{1}, \ldots, u_{d}$. We write $f \in R_{d}$ as

$$
f=\sum_{\mathbf{n} \in \mathbb{Z}^{d}} f_{\mathbf{n}} u^{\mathbf{n}}
$$

with $u^{\mathbf{n}}=u_{1}^{n_{1}} \cdots u_{d}^{n_{d}}$ and $f_{\mathbf{n}} \in \mathbb{Z}$ for all $\mathbf{n}=\left(n_{1}, \ldots, n_{d}\right) \in \mathbb{Z}^{d}$, where $f_{\mathbf{n}}=0$ for all but finitely many $\mathbf{n} \in \mathbb{Z}^{d}$.

If $\alpha$ is an algebraic $\mathbb{Z}^{d}$-action on a compact abelian group $X$, then the additively written dual group $M=\widehat{X}$ is a module over the ring $R_{d}$ with respect to the operation

$$
f \cdot a=\sum_{\mathbf{n} \in \mathbb{Z}^{d}} f_{\mathbf{n}} \widehat{\alpha}(\mathbf{n})(a)
$$

for $f \in R_{d}$ and $a \in M$, where $\widehat{\alpha}(\mathbf{n})$ denotes the automorphism of $\widehat{X}$ dual to $\alpha(\mathbf{n})$. The module $M=\widehat{X}$ is called the dual module of $\alpha$.

Conversely, if $M$ is a module over $R_{d}$, then we obtain an algebraic $\mathbb{Z}^{d}$-action $\alpha_{M}$ on $X_{M}=\widehat{M}$ by setting $\widehat{\alpha_{M}}(\mathbf{n})(a)=u^{\mathbf{n}} \cdot a$ for every $\mathbf{n} \in \mathbb{Z}^{d}$ and $a \in M$. Clearly, $M$ is the dual module of $\alpha_{M}$.

If $(X, \alpha)$ is an algebraic $\mathbb{Z}^{d}$-action and $\Lambda \subset \mathbb{Z}^{d}$ is a subgroup, then $\alpha^{\Lambda}$ will denote the restriction of $\alpha$ to $\Lambda$. For a $R_{d}$-module $M$, by $F(M)$ we denote the submodule consisting of all $m \in M$ such that $R_{d} \cdot m$ is finitely generated as an additive group. If $N \subset M$ is a submodule, then $N^{\perp} \subset X_{M}$ will denote the subgroup consisting of all $x$ such that $\chi(x)=1$ for all $\chi \in N$. By duality theory, the correspondence $N \mapsto N^{\perp}$ is an order-reversing bijection from the set of all submodules of $M$ to the set of all $\alpha_{M}$-invariant closed subgroups of $X_{M}$.

For an algebraic $\mathbb{Z}^{d}$-action $(X, \alpha)$, the topological entropy $h_{\text {top }}(\alpha)$ coincides with the metric entropy $h_{\lambda_{X}}(\alpha)$ (cf. [15). If an algebraic $\mathbb{Z}^{d}$-action has completely positive entropy, then it is Bernoulli (cf. [17]).

Recall that a prime ideal $\mathfrak{p} \subset R_{d}$ is associated with an $R_{d}$-module $M$ if $\mathfrak{p}=$ $\operatorname{ann}(a)=\left\{f \in R_{d}: f \cdot a=0_{M}\right\}$ for some $a \in M$. The set of prime ideals associated with $M$ will be denoted by $\operatorname{Asc}(M)$. We recall the following results from [15] and [19. 
Lemma 2.1. Let $d \geq 1$, and let $M$ be a countable $R_{d}$-module. Then $\alpha_{M}$ is mixing if and only if $\left\{u^{\mathbf{n}}-1: \mathbf{n} \in \mathbb{Z}^{d}\right\} \cap \mathfrak{p}=\{0\}$ for every $\mathfrak{p} \in A s c(M)$.

Lemma 2.2. Let $d \geq 1$, and let $M$ be a countable $R_{d}$-module such that the action $\alpha_{M}$ is mixing. Then:

(1) $\alpha_{M}$ has zero entropy if and only if every $\mathfrak{p} \in A s c(M)$ is non-principal.

(2) $\alpha_{M}$ has completely positive entropy if and only if every $\mathfrak{p} \in \operatorname{Asc}(M)$ is principal.

(3) If $M$ is Noetherian, then $\alpha_{M}$ has finite entropy if and only if every $\mathfrak{p} \in$ $\operatorname{Asc}(M)$ is non-zero.

The Krull dimension $\operatorname{kdim}(R)$ of a ring $R$ is the length $l$ of the longest chain

$$
\{0\}=\mathfrak{p}_{0} \subset \mathfrak{p}_{1} \subset \cdots \subset \mathfrak{p}_{l}
$$

of distinct prime ideals in $R$ (see [7, Chapter 8] for necessary background). The ring $R_{d}$ has Krull dimension $d+1$. If $\mathbb{K}$ is a field, then the transcendence degree $\operatorname{tdeg}(\mathbb{K})$ of $\mathbb{K}$ is the maximum number of elements in $\mathbb{K}$ that are algebraically independent over the prime subfield of $\mathbb{K}$. If $\mathfrak{p} \subset R_{d}$ is a prime ideal and $\mathbb{K}$ is the field of fractions of $R_{d} / \mathfrak{p}$, then $\operatorname{kdim}\left(R_{d} / \mathfrak{p}\right)=\operatorname{tdeg}(\mathbb{K})+1$ if $\operatorname{char}(\mathbb{K})=0$, and $\operatorname{kdim}\left(R_{d} / \mathfrak{p}\right)=\operatorname{tdeg}(\mathbb{K})$ if $\operatorname{char}(\mathbb{K})>0$.

For a compact abelian group $X$, we denote the connected component containing the identity by $X^{0}$. A connected compact abelian group $X$ is finite-dimensional if the dual group $\widehat{X}$ is isomorphic to a subgroup of $\mathbb{Q}^{n}$ for some $n \geq 1$.

Proposition 2.3. Let $M$ be a Noetherian $R_{2}$-module such that the action $\alpha_{M}$ is mixing and has zero entropy. Then $X_{M}^{0}$ is a finite-dimensional group.

Proof. Since the restriction of $\alpha_{M}$ to $X_{M}^{0}$ is also mixing and has zero entropy (cf. [19, Theorem 3.6]), it is enough to consider the case when $X_{M}$ is connected. Clearly, if there exists an $R_{2}$-module $N \supset M$ such that $X_{N}$ is finite-dimensional, then $X_{M}$ is finite-dimensional. Similarly, if there exists a submodule $M_{1} \subset M$ such that both $X_{M_{1}}$ and $X_{M / M_{1}}$ are finite-dimensional, then $X_{M}$ is finite-dimensional. As $M$ is a Noetherian module, there exists a Noetherian $R_{2}$-module $N \supset M$ and a finite sequence of submodules

$$
\{0\}=N_{0} \subset N_{1} \subset \cdots \subset N_{k}=N,
$$

such that for each $i \geq 1, N_{i+1} / N_{i}=R_{2} / \mathfrak{p}$ for some $\mathfrak{p} \in \operatorname{Asc}(M)$ (cf. 19, Corollary $6.3]$ ). Hence we may assume that $M=R_{2} / \mathfrak{p}$ for some prime ideal $\mathfrak{p} \subset R_{2}$. Let $\mathbb{K}$ denote the field of fractions of $R_{2} / \mathfrak{p}$. As $\mathfrak{p}$ is non-principal and $\operatorname{char}(\mathbb{K})=0$, $\operatorname{tdeg}(\mathbb{K})=\operatorname{kdim}\left(R_{2} / \mathfrak{p}\right)-1=0$. Since $R_{2} / \mathfrak{p}$ is a finitely generated ring, this shows that as an additive group $\mathbb{K}$ is isomorphic with $\mathbb{Q}^{n}$ for some $n \geq 1$, which proves the given assertion.

Our next lemma is a special case of Theorem 1.1. It follows from known results on invariant measures of algebraic $\mathbb{Z}^{d}$-actions and from a joining argument used in 12 and [13. We sketch a proof for completeness.

Lemma 2.4. Let $d>1$, and let $M$ and $N$ be countable $R_{d}$-modules with the following properties:

(1) $F(M)=M$.

(2) The actions $\alpha_{M}$ and $\alpha_{N}$ have zero entropy, and all lower rank subactions of $\alpha_{M}$ and $\alpha_{N}$ have completely positive entropy. 
Then every measurable equivariant map $f$ from $\left(X_{M}, \alpha_{M}\right)$ to $\left(X_{N}, \alpha_{N}\right)$ is an affine map.

Proof. We choose an arbitrary $m \in M$. Since $R_{d} \cdot m$ is finitely generated as an additive group and the action $\alpha_{M}$ is mixing, as an additive group $R_{d} \cdot m$ is isomorphic with $\mathbb{Z}^{k}$ for some $k \geq 0$. Hence $M$ is torsion free, and by duality $X_{M}$ is connected. In particular, the action $\alpha_{M}$ is mixing of all orders (cf. [21]). We also observe that $X_{R_{d} \cdot m}$ is isomorphic to a torus and $\left(X_{R_{d} \cdot m}, \alpha_{R_{d} \cdot m}\right)$ is a factor of $\left(X_{M}, \alpha_{M}\right)$. As every lower rank subaction of $\alpha_{M}$ has completely positive entropy, we deduce that either $X_{M}$ is trivial or $d=2$.

Define $i: X_{M} \rightarrow X_{M} \times X_{N}$ and a measure $\mu$ on $X_{M} \times X_{N}$ by $i(x)=(x, f(x))$, $\mu=i_{*}\left(\lambda_{X_{M}}\right)$. It is easy to see that $f$ is an affine map if and only if $\mu$ is affine, i.e. $\mu$ is a translate of the Haar measure on a closed subgroup of $X_{M} \times X_{N}$. An elementary harmonic analysis argument shows that $\mu$ is of this form if and only if $|\widehat{\mu}(\chi)|=0$ or 1 for every $\chi \in M \times N$, where $\widehat{\mu}$ is the Fourier transform of $\mu$ (cf. [19], p. 289).

We fix $\chi \in M \times N$ and set $P=R_{d} \cdot \chi$. Let $\pi$ denote the projection map from $X_{M} \times$ $X_{N}$ to $X_{M}, \pi^{\prime}$ denote the projection map from $X_{M} \times X_{N}$ to $X_{P}$, and $\mu_{P}$ denote the measure $\pi_{*}^{\prime}(\mu)$. Since $\pi$ is a measurable conjugacy from $\left(X_{M} \times X_{N}, \alpha_{M} \times \alpha_{N}, \mu\right)$ to $\left(X_{M}, \alpha_{M}, \lambda_{X_{M}}\right)$ and $\pi^{\prime}$ is a measurable factor map from $\left(X_{M} \times X_{N}, \alpha_{M} \times \alpha_{N}, \mu\right)$ to $\left(X_{P}, \alpha_{P}, \mu_{P}\right)$, with respect to the measure $\mu_{P}$ the action $\alpha_{P}$ is mixing of all orders, and every lower rank subaction of $\alpha_{P}$ has completely positive entropy. As $X_{P} / X_{P}^{0}$ is zero-dimensional, from [18] we deduce that the image of $\mu_{P}$ on $X_{P} / X_{P}^{0}$ is concentrated at a point. Hence $\mu_{P}$ is a translate of some $\alpha_{P}$-invariant measure $\nu$ on $X_{P}^{0}$. Since we only need to consider the case when $d=2$, and $\alpha_{P}$ has zero-entropy with respect to $\lambda_{X_{P}}$, by the previous proposition we may assume that $X_{P}^{0}$ is a finite-dimensional group. As all lower rank subactions of $\alpha_{P}$ have completely positive entropy with respect to the measure $\nu$, from [9, Theorem 1.3] we deduce that $\nu$ is an affine measure. This implies that $\mu_{P}$ is an affine measure and $|\widehat{\mu}(\chi)|=\left|\widehat{\mu_{P}}(\chi)\right| \in\{0,1\}$.

\section{Continuity AND Finiteness OF ENTROPy}

In this section we prove two lemmas which will be used in the proof of Theorem 1.1. We begin with some notation. Let $\left(Y, d_{Y}\right)$ be a compact metric space, and let $\beta$ be an action of $\mathbb{Z}^{d}$ on $Y$ by homeomorphisms. We denote the set of all $\beta$-invariant probability measures on $Y$ by $M_{\beta}(Y)$. With respect to the weak ${ }^{*}$ topology $M_{\beta}(Y)$ is a compact convex set. For any $\mu \in M_{\beta}(Y), h_{\mu}(\beta)$ will denote the entropy of the action $\beta$ with respect to the measure $\mu$. For $n \geq 1$, let $B_{n} \subset \mathbb{Z}^{d}$ denote the rectangle $\{0, \ldots, n\}^{d}$, and let $d_{n}$ denote the metric on $Y$ defined by

$$
d_{n}\left(y_{1}, y_{2}\right)=\max _{\mathbf{m} \in B_{n}} d_{Y}\left(\beta(\mathbf{m})\left(y_{1}\right), \beta(\mathbf{m})\left(y_{2}\right)\right) .
$$

For a closed set $C \subset Y$ and $\epsilon>0$, let $S_{n}(C, \epsilon)$ be the largest cardinality of an $\epsilon$-separating set in $C$ with respect to the metric $d_{n}$. We set

$$
S(C, \epsilon)=\limsup _{n \mapsto \infty} \frac{1}{n} \log S_{n}(C, \epsilon), \quad h_{\mathrm{top}}(\beta, C)=\lim _{\epsilon \mapsto 0} S(C, \epsilon) .
$$

Note that $h_{\mathrm{top}}(\beta, Y)$ is the topological entropy of the action $\beta$. For any $y \in$ $Y$ and $t>0$, let $A_{t}(y)$ denote the set of all $y^{\prime} \in Y$ with the property that 
$d_{n}\left(y, y^{\prime}\right) \leq t$ for all $n \geq 1$. The action $\beta$ is said to be asymptotically $h$-expansive if $\sup _{y \in Y} h_{\text {top }}\left(\beta, A_{t}(y)\right) \mapsto 0$ as $t \mapsto 0$.

We now recall a result from 16 which is a generalization of the well known fact that for an expansive $\mathbb{Z}^{d}$-action $\beta$, the map $\mu \mapsto h_{\mu}(\beta)$ is upper semicontinuous (see 16, Corollary 2.1 and Theorem 4.2]). Although in 16] the results are stated for $\mathbb{Z}$-actions, the proofs can easily be extended to $\mathbb{Z}^{d}$-actions for any $d \geq 1$.

Lemma 3.1. Let $Y$ be a compact metric space, and let $\beta$ be an asymptotically $h$-expansive $\mathbb{Z}^{d}$-action on $Y$. Then the map $\mu \mapsto h_{\mu}(\beta)$ is upper semicontinuous.

We note a simple consequence of the above result.

Lemma 3.2. Let $(Y, \beta)$ be an algebraic $\mathbb{Z}^{d}$-action with finite topological entropy. Then $\mu \mapsto h_{\mu}(\beta)$ is an upper semicontinuous map from $M_{\beta}(Y)$ to $\mathbb{R}$.

Proof. In view of the previous lemma it is enough to show that the action $(Y, \beta)$ is asymptotically $h$-expansive. Let $d_{Y}$ be a translation invariant metric on $Y$. For $t>0$ the closed set $Y_{t}=A_{t}(0)$ is invariant under $\beta$. Note that for any $y \in Y$ and $n \geq 1$, the map $x \mapsto x y$ is an isometry from $\left(Y_{t}, d_{n}\right)$ to $\left(A_{t}(y), d_{n}\right)$. This implies that for any $y \in Y$ and $t>0, h_{\text {top }}\left(\beta, A_{t}(y)\right)=h_{\text {top }}\left(\beta, Y_{t}\right)$. Suppose that $\lim \sup _{t \mapsto 0} h_{\text {top }}\left(\beta, Y_{t}\right)=c>0$. Since $h_{\text {top }}(\beta)$ is finite, we can choose $\epsilon>0$ such that $S(Y, \epsilon) \geq h_{\text {top }}(\beta)-c / 4$. We choose $0<t, \delta<\epsilon / 3$ such that $h_{\text {top }}\left(\beta, Y_{t}\right)>c / 2$ and $S\left(Y_{t}, \delta\right)>c / 3$. If $A_{1}$ is an $(n, \epsilon)$-separating set in $Y$ and $A_{2}$ is an $(n, \delta)$-separating set in $Y_{t}$, then $A_{1} \cdot A_{2}$ is an $(n, \delta)$-separating set in $Y$. Hence

$$
S_{n}(Y, \epsilon) \cdot S_{n}\left(Y_{t}, \delta\right) \leq S_{n}(Y, \delta)
$$

which implies that $h_{\text {top }}(\beta)-c / 4+c / 3 \leq h_{\text {top }}(\beta)$. This contradiction shows that the action $\beta$ is asymptotically $h$-expansive.

We now prove a lemma on finiteness of entropy of rank $d-1$ subactions. It is easy to construct examples of zero-entropy algebraic $\mathbb{Z}^{d}$-actions with a Noetherian dual module which admit rank $d-1$ subactions with infinite entropy. For example, if $A$ is the shift automorphism on $\mathbb{T}^{\mathbb{Z}}$ and $B=\mathrm{Id}$, then the $\mathbb{Z}^{2}$-action generated by $A$ and $B$ has zero entropy, but the cyclic action generated by $A$ has infinite entropy. We show that this situation does not occur if the action is mixing.

Lemma 3.3. Let $d>1$, and let $M$ be a Noetherian $R_{d}$-module such that the action $\alpha_{M}$ is mixing and has zero entropy. Then for any subgroup $\Lambda \subset \mathbb{Z}^{d}$ with $\operatorname{rank}(\Lambda)=d-1$, the action $\alpha_{M}^{\Lambda}$ has finite entropy.

Proof. Let $C_{d}$ denote the class of Noetherian $R_{d}$-modules $M$ with the property that $\alpha_{M}$ is a mixing action with zero-entropy and that all rank $d-1$ subactions of $\alpha_{M}$ have finite entropy. Since any factor of $\alpha_{M}$ also has these properties, the class $C_{d}$ is closed under taking submodules. If $M \subset N$ is a submodule such that $M$ and $N / M$ lie in $C_{d}$, then from the entropy addition formula (cf. 19, Theorem 14.1]) it follows that $N \in C_{d}$. Now as in the proof of Proposition 2.3, we may assume that $M=R_{d} / \mathfrak{p}$ for some prime ideal $\mathfrak{p} \subset R_{d}$.

Let $\Lambda_{0} \subset \mathbb{Z}^{d}$ denote the subgroup consisting of all $\mathbf{n}=\left(n_{1}, \ldots, n_{d}\right)$ with $n_{d}=0$. We choose an injective endomorphism $\phi: \mathbb{Z}^{d} \rightarrow \mathbb{Z}^{d}$ such that $\phi\left(\Lambda_{0}\right)=\Lambda$, and define a $\mathbb{Z}^{d}$-action $\alpha^{0}$ on $X_{M}$ by setting $\alpha^{0}(\mathbf{n})=\alpha(\phi(\mathbf{n}))$ for all $\mathbf{n} \in \mathbb{Z}^{d}$. Clearly, the action $\alpha^{0}$ is mixing and has zero entropy. Replacing $M$ by the dual module of the action $\alpha^{0}$ if necessary, we may assume that $\Lambda=\Lambda_{0}$. 
Let $R_{d-1} \subset R_{d}$ denote the subring $\mathbb{Z}\left[u^{ \pm 1}, \ldots, u^{ \pm d-1}\right]$, and let $R$ denote the image of $R_{d-1}$ in $R_{d} / \mathfrak{p}$. Then as an $R_{d-1}$-module $R$ is isomorphic with $R_{d-1} / R_{d-1} \cap \mathfrak{p}$. Let $\mathbb{F}$ denote the field of fractions of $R$, and let $\mathbb{K}$ denote the field of fractions of $R_{d} / \mathfrak{p}$.

We claim that the $\Lambda$-action $\alpha_{R}$ has finite entropy. By Lemma 2.2 it is enough to show that $R_{d-1} \cap \mathfrak{p} \neq\{0\}$. This is obvious if $\operatorname{char}(\mathbb{K})>0$. If $\operatorname{char}(\mathbb{K})=0$, then $\operatorname{tdeg}(\mathbb{K})=\operatorname{kdim}\left(R_{d} / \mathfrak{p}\right)-1 \leq d-2$, as $\mathfrak{p}$ is a non-principal prime ideal. Since the transcendence degree of the field of fractions of $R_{d-1}$ is $d-1$, the map from $R_{d-1}$ to $R_{d} / \mathfrak{p} \subset \mathbb{K}$ induced by the inclusion map $i: R_{d-1} \rightarrow R_{d}$ is not injective. This shows that $R_{d-1} \cap \mathfrak{p} \neq\{0\}$, which proves the claim. Note that for any finitely generated $R_{d-1}$-module $N \subset \mathbb{F}$ we can choose $r_{0} \in R$ such that $r_{0} \cdot N \subset R$. Hence $N$ is isomorphic with a submodule of $R$, i.e. $\alpha_{N}$ is an algebraic factor of $\alpha_{R}$. In particular, $h_{\mathrm{top}}\left(\alpha_{N}\right) \leq h_{\mathrm{top}}\left(\alpha_{R}\right)$. We choose an increasing sequence of $R_{d-1^{-}}$ submodules $N_{1} \subset N_{2} \subset \cdots \subset \mathbb{F}$ such that $\bigcup_{j} N_{j}=\mathbb{F}$. Since $N_{j}^{\perp} \mapsto 0_{X_{\mathbb{F}}}$ as $j \mapsto \infty$, from [19, Lemma 13.6] it follows that

$$
h_{\mathrm{top}}\left(\alpha_{\mathbb{F}}\right)=\lim _{j \mapsto \infty} h_{\mathrm{top}}\left(\alpha_{N_{j}}\right) \leq h_{\mathrm{top}}\left(\alpha_{R}\right)<\infty .
$$

Now we consider the cases $[\mathbb{K}: \mathbb{F}]<\infty$ and $[\mathbb{K}: \mathbb{F}]=\infty$ separately. In the former case, the $R_{d-1}$-module $\mathbb{K}$ is a direct sum of finitely many copies of $\mathbb{F}$. Since $h_{\text {top }}\left(\alpha_{\mathbb{F}}\right)<\infty$ and $M=R_{d} / \mathfrak{p}$ is an $R_{d-1}$-submodule of $\mathbb{K}$, the action $\alpha_{M}^{\Lambda}$ has finite entropy. In the latter case the element $u_{d}$ is not algebraic over $\mathbb{F}$. This can happen only if $\mathfrak{p}$ is contained in $R_{d-1}$. It is easy to see that $R_{d-1} \cap \mathfrak{p}$ is the only prime ideal associated to the $R_{d-1}$-module $M$. Since $\mathfrak{p}$ is a non-principal ideal, by Lemma 2.2 the action $\alpha_{M}^{\Lambda}$ has zero entropy.

\section{RIGIDITY OF EQUIVARIANT MAPS}

Recall that a valuation on a ring $R$ is a map $v: R \rightarrow \mathbb{R} \cup\{\infty\}$ satisfying the following conditions:

(1) $v(1)=0$ and $v(x)=\infty$ if and only if $x=0$,

(2) $v(x y)=v(x)+v(y)$,

(3) $v(x+y) \geq \operatorname{Min}\{v(x), v(y)\}$.

Let $\mathfrak{p} \subset R_{d}$ be a prime ideal. For a valuation $v$ on $R_{d} / \mathfrak{p}$ we define a homomorphism $\phi_{v}: \mathbb{Z}^{d} \rightarrow \mathbb{R}$ by $\phi_{v}(\mathbf{n})=v\left(u^{\mathbf{n}}\right)$. The valuation $v$ is said to be discrete if the image of $\phi_{v}$ is a non-trivial discrete subgroup of $\mathbb{R}$. It is easy to see that $v$ is discrete if and only if the subgroup $\Lambda_{v}=\left\{\mathbf{n}: \phi_{v}(\mathbf{n})=0\right\} \subset \mathbb{Z}^{d}$ has rank $d-1$.

The following result characterizes all prime ideals $\mathfrak{p} \subset R_{d}$ with the property that $R_{d} / \mathfrak{p}$ admits a discrete valuation. For a proof see [5, Theorem 2.4] and [6, Corollary 1 and Theorem 8.1].

Lemma 4.1. Let $d \geq 1$, and let $\mathfrak{p} \subset R_{d}$ be a prime ideal. Then $R_{d} / \mathfrak{p}$ admits a discrete valuation if and only if it is not finitely generated as an additive group.

Definition. Let $d \geq 1$, and let $M$ be a countable $R_{d}$-module. A closed subgroup $H \subset X_{M}$ is $\alpha_{M}$-shrinking if there exists an $\mathbf{n}_{H} \in \mathbb{Z}^{d}$ such that

$$
\bigcap_{j \geq 1} \alpha_{M}\left(j \mathbf{n}_{H}\right)(H)=\{0\}
$$

and $\alpha_{M}(\mathbf{n})(H)=H$ whenever $\left\langle\mathbf{n}, \mathbf{n}_{H}\right\rangle=0$, where $\langle.,$.$\rangle denotes the standard inner$ product in $\mathbb{R}^{d}$. 
The smallest closed subgroup of $X_{M}$ which contains all $\alpha_{M}$-shrinking subgroups will be denoted by $X_{M}^{s}$. It is easy to see that if $M, N$ are $R_{d}$-modules and $\theta$ : $X_{M} \rightarrow X_{N}$ is a continuous $\mathbb{Z}^{d}$-equivariant homomorphism, then $\theta\left(X_{M}^{s}\right) \subset X_{N}^{s}$.

Proposition 4.2. Let $d \geq 1$, let $M$ be a countable $R_{d}$-module and let $F(M)$ be as defined before. Then $X_{M}^{s}=F(M)^{\perp}$.

Proof. First we consider the case when $F(M)=\{0\}$. If the above assertion is not true, then $X_{M}^{s}=M_{1}^{\perp}$ for some non-zero submodule $M_{1}$ of $M$. In that case we choose $\mathfrak{p} \in \operatorname{Asc}\left(M_{1}\right)$ and $m_{0} \in M_{1}$ with $\operatorname{ann}\left(m_{0}\right)=\mathfrak{p}$. Since $F(M)=\{0\}, R_{d} / \mathfrak{p}$ is not finitely generated as an additive group. By the previous lemma there exists a discrete valuation $v$ on $R_{d} / \mathfrak{p}$. We set

$$
R=\left\{p \in R_{d}: v(p) \geq 0\right\}, M_{0}=\left\{p \cdot m_{0}: v(p) \geq 1\right\} .
$$

It is easy to see that $R$ is an Noetherian ring and $M_{0}$ is an $R$-module.

For any $A \subset M$, let $\bar{A}$ denote the $R_{d}$-submodule generated by $A$. We define a partial order on the set of all $R$-submodules of $M$ by setting $N \leq N^{\prime}$ if $N \subset N^{\prime}$ and $N^{\prime} \cap \bar{N}=N$. It is easy to see that for any totally ordered subset $C$, the $R$-module $\cup\{N: N \in C\}$ is an upper bound for $C$. By Zorn's lemma there exists a maximal element $N_{0}$ in $\left\{N: N \geq M_{0}\right\}$. We claim that $\overline{N_{0}}=M$. Suppose this is not the case. Let $m$ be an element of $M-\overline{N_{0}}$. Since $R$ is Noetherian, the $R$-module $R \cdot m \cap \overline{N_{0}}$ is finitely generated. We choose $\mathbf{n} \in \mathbb{Z}^{d}$ with $v(\mathbf{n})<0$, and observe that

$$
\overline{N_{0}}=\bigcup_{j \geq 1} u^{j \mathbf{n}} \cdot N_{0}
$$

Let $B$ be a finite set which generates $R \cdot m \cap \overline{N_{0}}$ as an $R$-module. We find $k \geq 1$ such that $u^{-k \mathbf{n}} \cdot b \in N_{0}$ for all $b \in B$. If $m^{\prime}=u^{-k \mathbf{n}} \cdot m$, then $\left(R \cdot m^{\prime}+N_{0}\right) \cap \overline{N_{0}}=$ $R \cdot m^{\prime} \cap \overline{N_{0}}+N_{0}=N_{0}$, which contradicts the maximality of $N_{0}$ and proves the claim.

Now from the above claim and duality theory we deduce that

$$
\bigcap_{j \geq 1} \alpha(j \mathbf{n})\left(N_{0}^{\perp}\right)=\{0\}
$$

i.e. $N_{0}^{\perp}$ is an $\alpha$-shrinking subgroup of $X_{M}$. Hence $N_{0} \supset M_{1}$. As $N_{0} \cap \overline{M_{0}}=M_{0}$, we deduce that $m_{0} \in M_{0}$, i.e. $p \cdot m_{0}=m_{0}$ for some $p \in R_{d}$ with $v(p) \geq 1$. Since $0=v(1) \geq \operatorname{Min}\{v(p), v(1-p)\}$, we arrive at a contradiction. This completes the proof of the given assertion in the special case considered above.

Now we consider the general case. For $a \in F(M)$, let $M_{a}$ denote the $R_{d^{-}}$ submodule generated by $a$, and let $\pi_{a}: X_{M} \rightarrow X_{M_{a}}$ denote the dual of the inclusion map $i: M_{a} \rightarrow M$. Since $M_{a}$ is finitely generated as an additive group, $X_{M_{a}}$ is isomorphic with $\mathbb{T}^{n} \times F$, where $\mathbb{T}^{n}$ is a torus and $F$ is a finite abelian group. Hence $X_{M_{a}}$ does not admit arbitrarily small non-trivial subgroups. In particular, $X_{M_{a}}^{s}=\{0\}$. Since $\pi_{a}$ is a continuous $\mathbb{Z}^{d}$-equivariant homomorphism for every $a \in F(M)$, this shows that

$$
X_{M}^{s} \subset \bigcap_{a \in F(M)} \operatorname{Ker}\left(\pi_{a}\right)=F(M)^{\perp}
$$


Let $\theta: X_{M / F(M)} \rightarrow X_{M}$ denote the dual of the projection map from $M$ to $M / F(M)$. Since $F(M / F(M))=\{0\}, X_{M / F(M)}^{s}=X_{M / F(M)}$. As $\theta$ is a continuous $\mathbb{Z}^{d}$-equivariant homomorphism, this implies that $F(M)^{\perp}=\theta\left(X_{M / F(M)}\right)=$ $\theta\left(X_{M / F(M)}^{s}\right) \subset X_{M}^{s}$.

A measurable map $f: X \rightarrow Y$ between compact abelian groups is a constant if there exists $c \in Y$ such that $f(x)=c$ almost everywhere with respect to $\lambda_{X}$. For a measurable map $f: X \rightarrow Y$, by $C(f)$ we denote the set of all $h \in X$ with the property that the map $x \mapsto f(x+h)-f(x)$ is a constant. We note the following elementary fact.

Proposition 4.3. Let $f: X \rightarrow Y$ be a measurable map between compact abelian groups. Then $C(f)$ is a closed subgroup of $X$, and $f$ is an affine map if and only if $C(f)=X$.

Proof. By duality theory, the map $f$ is affine if and only if $\chi \circ f$ is an affine map for every $\chi \in \widehat{Y}$. Since $C(f)=\bigcap_{\chi} C(\chi \circ f)$, without loss of generality we may assume that $Y=\mathbb{T}$. It is easy to see that $C(f) \subset X$ is a subgroup, and $C(f)=X$ whenever $f$ is an affine map. As the translation action of $X$ on $L^{2}(X)$ is continuous, and the space of constant maps from $X$ to $\mathbb{T}$ is a closed subset of $L^{2}(X), C(f)$ is closed. Since all eigenvalues of the translation action are of the form $c \cdot \chi$ for some $c \in \mathbb{C}$ and $\chi \in \widehat{X}, C(f)=X$ only if $f$ is an affine map.

Proof of Theorem 1.1. Let $M$ and $N$ be the dual modules of $\alpha$ and $\beta$, respectively. For any $a \in N$, let $Y_{a} \subset Y$ denote the dual of $R_{d} \cdot a$, and let $\beta_{a}$ denote the $\mathbb{Z}^{d}$-action on $Y_{a}$ induced by $\beta$. If $\pi_{a}$ denotes the factor map from $(Y, \beta)$ to $\left(Y_{a}, \beta_{a}\right)$, then it is easy to see that the map $f$ is affine if and only if $\pi_{a} \circ f$ is affine for every $a \in N$. Since the dual module of each $\beta_{a}$ is Noetherian, without loss of generality we may assume that $N$ is a Noetherian $R_{d}$-module.

We define a map $q: X \times X \rightarrow Y$ by

$$
q\left(x, x^{\prime}\right)=f\left(x+x^{\prime}\right)-f\left(x^{\prime}\right) .
$$

For $\mu \in M(X)$, let $\bar{\mu}$ denote the measure $q_{*}\left(\mu \times \lambda_{X}\right) \in M(Y)$. It is easy to see that $q$ is a $\mathbb{Z}^{d}$-equivariant map, and $\overline{\alpha(\mathbf{m})(\mu)}=\beta(\mathbf{m})(\bar{\mu})$ for all $\mu \in M(X)$ and $\mathbf{m} \in \mathbb{Z}^{d}$. We claim that $\bar{\mu} \mapsto \delta_{0_{Y}}$ as $\mu \mapsto \delta_{0_{X}}$. Let $\widehat{\bar{\mu}}$ denote the Fourier transform of $\bar{\mu}$. We fix a non-zero character $\chi \in N$, and note that

$$
\widehat{\bar{\mu}}(\chi)=\int \chi \mathrm{d} \bar{\mu}=\int \chi \circ q\left(x, x^{\prime}\right) \mathrm{d} \mu(x) \mathrm{d} \lambda_{X}\left(x^{\prime}\right) .
$$

We set $q_{\chi}=\chi \circ q$ and $f_{\chi}=\chi \circ f$. For $x \in X$, we denote the $L^{1}$-norm of the function $x^{\prime} \mapsto q_{\chi}\left(x, x^{\prime}\right)$ by $P(x)$. Since the translation action of $X$ on $L^{1}(X)$ is strongly continuous, and

$$
\left|q_{\chi}\left(x, x^{\prime}\right)\right|=\left|f_{\chi}\left(x+x^{\prime}\right) \cdot \overline{f_{\chi}\left(x^{\prime}\right)}\right| \leq\left|f_{\chi}\left(x+x^{\prime}\right)-f_{\chi}\left(x^{\prime}\right)\right|,
$$

it follows that $P: X \rightarrow \mathbb{R}$ is a continuous function with $P\left(0_{X}\right)=0$. Since $|\widehat{\bar{\mu}}(\chi)| \leq$ $\int P(x) \mathrm{d} \mu(x), \widehat{\bar{\mu}}(\chi) \mapsto 0$ as $\mu \mapsto \delta_{0_{X}}$. This proves the claim. 
We fix an $\alpha$-shrinking subgroup $H \subset X$ and define

$$
\Lambda=\left\{\mathbf{n} \in \mathbb{Z}^{d}:\left\langle\mathbf{n}, \mathbf{n}_{H}\right\rangle=0\right\} .
$$

Since $\operatorname{rank}(\Lambda)=\mathrm{d}-1$, by Lemma 3.3 the action $\beta^{\Lambda}$ has finite entropy. For $j \geq 0$, let $\mu_{j}$ denote the Haar measure on $\alpha\left(j \mathbf{n}_{H}\right)(H)$. As $\mu_{0}=\lambda_{H}$ is invariant under the action $\alpha^{\Lambda}$, the measure $\overline{\mu_{0}}$ is invariant under $\beta^{\Lambda}$. Since $\mu_{j} \mapsto \delta_{0_{X}}$ as $j \mapsto \infty$, from the above claim we deduce that $\overline{\mu_{j}} \mapsto \delta_{0_{Y}}$ as $j \mapsto \infty$. We note that $\beta(\mathbf{n})$ commutes with the action $\beta^{\Lambda}$ for all $\mathbf{n} \in \mathbb{Z}^{d}$. This implies that the $\Lambda$-actions $\left(Y, \beta^{\Lambda}, \overline{\mu_{0}}\right)$ and $\left(Y, \beta^{\Lambda}, \overline{\mu_{j}}\right)$ are measurably conjugate for every $j \geq 1$. By Lemma 3.2 .

$$
h_{\overline{\mu_{0}}}\left(\beta^{\Lambda}\right)=\lim _{j \mapsto \infty} h_{\overline{\mu_{j}}}\left(\beta^{\Lambda}\right)=0 .
$$

We set $X_{1}=\left(X, \alpha^{\Lambda}, \lambda_{H}\right), X_{2}=\left(X, \alpha^{\Lambda}, \lambda_{X}\right)$ and $Y_{1}=\left(Y, \beta^{\Lambda}, \overline{\mu_{0}}\right)$. Since $q$ : $X_{1} \times X_{2} \rightarrow Y_{1}$ is a factor map and $Y_{1}$ has zero-entropy, the map $q$ is measurable with respect to the Pinsker algebra of $X_{1} \times X_{2}$. As the Pinsker algebra of the product of two measure preserving $\mathbb{Z}^{d}$-actions is the product of their Pinsker algebras (cf. 11. Theorem 4]), and since $X_{2}$ has completely positive entropy, there exists a measurable map $g: X \rightarrow Y$ such that $q\left(x, x^{\prime}\right)=g(x)$ almost everywhere with respect to $\lambda_{H}$. An application of Fubini's theorem shows that for $\lambda_{H}$-a.e. $h \in H$, the map $x^{\prime} \mapsto q\left(h, x^{\prime}\right)$ is a constant. As our initial choice of $H$ was arbitrary, from the previous proposition we deduce that $X_{M}^{s} \subset C(f)$. Hence the map $q^{1}: X \times X \rightarrow Y$ defined by

$$
q^{1}\left(x, x^{\prime}\right)=f\left(x+x^{\prime}\right)-f(x)-f\left(x^{\prime}\right)
$$

is invariant under the translation action of $X_{M}^{s} \times X_{M}^{s}$ on $X \times X$. Let $\pi$ denote the projection map from $X \times X$ to $Z=X / X_{M}^{s} \times X / X_{M}^{s}$, and let $q^{2}: Z \rightarrow Y$ denote the measurable map satisfying $q^{1}=q^{2} \circ \pi$. If $\alpha_{Z}$ denotes the $\mathbb{Z}^{d}$-action on $Z$ induced by $\alpha$, then by Proposition 4.2 the dual module of $\alpha_{Z}$ is isomorphic with $F(M) \times F(M)$. Since $q^{2}$ is a $\mathbb{Z}^{d}$-equivariant map, by Lemma 2.4 both $q^{2}$ and $q^{2} \circ \pi=q^{1}$ are affine maps. As $q^{1}\left(x, x^{\prime}\right)=q^{1}\left(x^{\prime}, x\right)$, this implies that for any $t \in X$, $q^{1}\left(x+t, x^{\prime}\right)=q^{1}\left(x, x^{\prime}+t\right)$ almost everywhere with respect to $\lambda_{X} \times \lambda_{X}$. Hence for any $t \in X$,

$$
f(x+t)+f\left(x^{\prime}\right)=f\left(x+t+x^{\prime}\right)-q^{1}\left(x+t, x^{\prime}\right)=f(x)+f\left(x^{\prime}+t\right),
$$

from which we deduce that $X=C(f)$. By the previous proposition $f$ is an affine map.

An algebraic $\mathbb{Z}^{d}$-action $\alpha$ is prime if the dual module of $\alpha$ is of the form $R_{d} / \mathfrak{p}$ for some prime ideal $\mathfrak{p} \subset R_{d}$. Prime actions can be viewed as building blocks of algebraic $\mathbb{Z}^{d}$-actions (see [19, Figure 1]). From duality theory it follows that $X_{R_{d} / \mathfrak{p}}$ is connected if $\mathfrak{p} \cap \mathbb{Z}=\{0\}$, and is zero-dimensional if $\mathfrak{p} \cap \mathbb{Z} \neq\{0\}$. In the zero-dimensional case in [3] and [8] it was shown that any measurable factor map between mixing zero-entropy prime actions is an affine map. As an application of Theorem 1.1 we now extend this result to all mixing zero-entropy prime actions.

Corollary 4.4. Let $d>1$, and let $\mathfrak{p}, \mathfrak{q} \subset R_{d}$ be prime ideals such that the actions $\alpha_{R_{d} / \mathfrak{p}}$ and $\alpha_{R_{d} / \mathfrak{q}}$ are mixing and have zero-entropy. Then any measurable factor map from $\left(X_{R_{d} / \mathfrak{p}}, \alpha_{R_{d} / \mathfrak{p}}\right)$ to $\left(X_{R_{d} / \mathfrak{q}}, \alpha_{R_{d} / \mathfrak{q}}\right)$ is an affine map. In particular, the actions $\alpha_{R_{d} / \mathfrak{p}}$ and $\alpha_{R_{d} / \mathfrak{q}}$ are measurably conjugate if and only if $\mathfrak{p}=\mathfrak{q}$. 
Proof. If $d_{1} \leq d$ and $\phi: \mathbb{Z}^{d_{1}} \rightarrow \mathbb{Z}^{d}$ is an injective homomorphism, we define an algebraic $\mathbb{Z}^{d_{1}}$-action $\alpha^{\phi}$ on $X_{R_{d} / \mathfrak{p}}$ by setting $\alpha^{\phi}(\mathbf{m})=\alpha_{R_{d} / \mathfrak{p}}(\phi(\mathbf{m}))$ for all $\mathbf{m} \in \mathbb{Z}^{d_{1}}$. For any such $\phi$, let $\phi_{*}: R_{d_{1}} \rightarrow R_{d}$ denote the induced ring homomorphism, and let $\mathfrak{p}_{\phi} \subset R_{d_{1}}$ denote the prime ideal $\phi_{*}^{-1}(\mathfrak{p})$. If $M_{1}$ denotes the dual module of $\alpha^{\phi}$, then it is easy to see that $\operatorname{Asc}\left(M_{1}\right)=\left\{\mathfrak{p}_{\phi}\right\}$. By Lemma 2.2 the action $\alpha^{\phi}$ has zero-entropy if $\mathfrak{p}_{\phi}$ is non-principal, and it has completely positive entropy if $\mathfrak{p}_{\phi}$ is principal. Let $d_{0}$ be the smallest integer such that for some injective homomorphism $\phi_{0}: \mathbb{Z}^{d_{0}} \rightarrow \mathbb{Z}^{d}$ the ideal $\mathfrak{p}_{\phi_{0}} \subset R_{d_{0}}$ is non-principal. We set $\Lambda=\phi\left(\mathbb{Z}^{d_{0}}\right)$, and note that the action $\alpha_{R_{d} / \mathfrak{p}}^{\Lambda}$ has zero-entropy but all lower rank subactions of $\alpha_{R_{d} / \mathfrak{p}}^{\Lambda}$ have completely positive entropy. Since $\left(X_{R_{d} / \mathfrak{q}}, \alpha_{R_{d} / \mathfrak{q}}\right)$ is a factor of $\left(X_{R_{d} / \mathfrak{p}}, \alpha_{R_{d} / \mathfrak{p}}\right)$, the same is true for the action $\alpha_{R_{d} / \mathfrak{q}}^{\Lambda}$. By Theorem 1.1, $f$ is an affine map. Hence if $f$ is a measurable conjugacy, then the actions $\alpha_{R_{d} / \mathfrak{p}}$ and $\alpha_{R_{d} / \mathfrak{q}}$ are algebraically conjugate. By duality, this happens only if $R_{d} / \mathfrak{p}$ and $R_{d} / \mathfrak{q}$ are isomorphic $R_{d^{-}}$ modules, i.e. $\mathfrak{p}=\mathfrak{q}$.

\section{REFERENCES}

[1] S. Bhattacharya, Zero entropy algebraic $\mathbf{Z}^{d}$-actions that do not exhibit rigidity, Duke Math. J., vol. 116, 471-476, 2003. MR1958095 (2004c:37010)

[2] S. Bhattacharya, Higher order mixing and rigidity of algebraic actions on compact abelian groups, Israel J. Math., vol. 137, 211-221, 2003. MR.2013357(2004g:37030)

[3] S. Bhattacharya and K. Schmidt, Homoclinic points and isomorphism rigidity of algebraic $\mathbf{Z}^{d}$-actions on zero dimensional compact abelian groups, Israel J. Math., vol. 137, 189-209, 2003. MR2013356 (2004g:37029)

[4] S. Bhattacharya and T. Ward, Finite entropy characterizes topological rigidity, Ergodic Theory Dynam. Systems, vol. 25, 365-373, 2005. MR2129101 (2006g:37003)

[5] R. Bieri and J.R.J. Groves, The geometry of the set of characters induced by valuations, J. Reine Angew. Math., vol. 347, 168-195, 1984. MR733052 (86c:14001)

[6] R. Bieri and R. Strebel, Valuations and finitely presented metabelian groups, Proc. London Math. Soc. (3), vol. 41, 439-464, 1980. MR591649 (81j:20080)

[7] D. Eisenbud, Commutative algebra with a view toward algebraic geometry, Graduate Texts in Mathematics 150, Springer-Verlag, New York, 1995. MR1322960 (97a:13001)

[8] M. Einsiedler, Isomorphism and measure rigidity for algebraic actions on zero-dimensional groups, Monatsh. Math., vol. 144, 39-69, 2005. MR2109928 (2005i:37008)

[9] M. Einsiedler and E. Lindenstrauss, Rigidity properties of $\mathbb{Z}^{d}$-actions on tori and solenoids, Electron. Res. Announc. Amer. Math. Soc., vol. 9, 99-110, 2003. MR2029471 (2005d:37007)

[10] M. Einsiedler and T. Ward, Isomorphism rigidity in entropy rank two, Israel J. Math., vol. 147, 269-284, 2005. MR2166364 (2006m:28022)

[11] E. Glasner, J.-P. Thouvenot and B. Weiss, Entropy theory without a past, Ergodic Theory Dynam. Systems, vol. 20, 1355-1370, 2000. MR.1786718 (2001h:37011)

[12] A. Katok, S. Katok and K. Schmidt, Rigidity of measurable structure for $\mathbb{Z}^{d}$-actions by automorphisms of a torus, Comment. Math. Helv., vol. 77, 718-745, 2002. MR1949111 (2003h:37007)

[13] B. Kitchens and K. Schmidt, Isomorphism rigidity of irreducible algebraic $\mathbb{Z}^{d}$-actions, Invent. Math., vol. 142, 559-577, 2000. MR1804161 (2001j:37004)

[14] D. Lind, The structure of skew products with ergodic group automorphisms, Israel J. Math., vol. 28, 205-248, 1977. MR0460593 (57:586)

[15] D. Lind, K. Schmidt and T. Ward, Mahler measure and entropy for commuting automorphisms of compact groups, Invent. Math., vol. 101, 593-629, 1990. MR.1062797 (92j:22013)

[16] M. Misiurewicz, Topological conditional entropy, Studia Math., vol. 55, 175-200, 1976. MR0415587 (54:3672)

[17] D. Rudolph and K. Schmidt, Almost block independence and Bernoullicity of $\mathbb{Z}^{d}$-actions by automorphisms of compact abelian groups, Invent. Math., vol. 120, 455-488, 1995. MR:1334481 (96d:22004) 
[18] K. Schmidt, Invariant measures for certain expansive $\mathbb{Z}^{2}$-actions, Israel J. Math., vol. 90, 295-300, 1995. MR1336327 (96c:28028)

[19] K. Schmidt, Dynamical systems of algebraic origin, Progress in Mathematics, 128, Birkhäuser Verlag, Basel, 1995. MR1345152 (97c:28041)

[20] K. Schmidt, Algebraic $\mathbb{Z}^{d}$-actions, Pacific Institute for the Mathematical Sciences Distinguished Chair Lecture Notes (electronic publication), University of Victoria, BC, 2002.

[21] K. Schmidt and T. Ward, Mixing automorphisms of compact groups and a theorem of Schlickewei, Invent. Math., vol. 111, 69-76, 1993. MR1193598(95c:22011)

School of Mathematics, Tata Institute of Fundamental Research, Mumbai 400005, INDIA

E-mail address: siddhart@math.tifr.res.in 